\title{
Effects of darkness, constant illumination, and synchronized photic stimulation on auditory sensitivity to pulsed tones'
}

\author{
J. A. SHERIDAN ${ }^{2}$, R. S. CIMBALO ${ }^{3}$, J. A. SILLS ${ }^{4}$, AND E. A. ALLUISI \\ UNIVERSITY OF LOUISVILLE
}

Pulsed-tone thresholds at five frequencies $(250,500,1000$, 2000 , and $6000 \mathrm{~Hz}$ ) were obtained from $20 \mathrm{Ss}$ under three conditions of visual surround: darkness, normal ambient itlumination, and relatively high-intensity-tone-synchronized photic stimulation. Auditory sensitivity to the highest frequency was lowered by visual stimulation of both types.

In an 1888 study of audio-visual interaction, Urbantschitsch found that pure-tone auditory stimulation (produced with tuning forks) improved color sensitivity. Other researchers have confirmed that the presence of sound improves visual sensitivity (e.g., Moruyama, 1961; Smith, 1965; Symonds, 1963).

The effects of visual stimulation on auditory sensitivity are less clear. Facilitation has been reported by some researchers (e.g., Gregg \& Brogden, 1952; Moruyama, 1961; Thompson et al, 1958), inhibition by others (Markov, 1960), and no intersensory effects by still other researchers (Gulick \& Smith, 1959; Rubinstein \& Loeb, 1955).

The present study was designed to measure the effects of visual stimulation on self-determined auditory thresholds for pulsed tones at five frequencies. The effects of darkness and of synchronized photic stimulation were compared relative to a control condition of normal, comfortable, constant, general illumination. Method

The auditory signal (and the signal for the synchronized photic stimulation) was generated with a beat frequency oscillator (GR 1304-B). An electronic switch (Grason-Stadler 829C), controlled by an interval timer (Grason-Stadler 471-1) and power supply (GrasonStadler 477-2), provided two 0.25-sec. pulses per sec., each with $10-\mathrm{msec}$. rise and decay times. This signal was fed to a mixer and split. One part of the split signal was put into the amplifying circuit of a Békésy audiometer $(E-800)$; the output went to S's right ear via a headset equipped with headphones (TDH-39) enclosed in Maico Auraldomes. The audiometer was calibrated and adjusted to read in $\mathrm{AB}$ (SPL). The second half of the split signal was used to trigger the synchronized photic stimulation. The signal was put first into one channel of a speech audiometer (Grason-Stadler 162), and from there into an amplifier (McIntosh MC-30) and appropriate transformers which increased its voltage to a level sufficient for reliable activation of preheated flourescent light sources ( two $15-\mathrm{w}, 8 / \mathrm{CW}$ bulbs).
Twenty Ss (10 males and 10 females) were obtained from psychology student volunteers at the University of Louisville. No $\mathrm{S}$ showed more than a 5-dB hearing loss at octave-step pure-tone test frequencies between 250 and $4000 \mathrm{cps}$, or at $6000 \mathrm{cps}$.

Following audiometer screening, each S participated in a practice session and three testing sessions. During all sessions $\mathrm{S}$ occupied one side of an IAC standard audiometer testing suite; $\mathrm{E}$ occupied the other side. In the practice session $S$ tracked the threshold of a monaurally presented $1500-\mathrm{Hz}$ pulsed tone for $2 \mathrm{~min}$. During each of the three testing sessions $\mathrm{S}$ tracked for $1 \mathrm{~min}$. at each of five frequencies: $250,500,1000$, 2000 , and $6000 \mathrm{~Hz}$; the three sessions differed in terms of the visual stimulation presented concurrently with the auditory signals. These three conditions will be referred to as normal ambient lighting (NAL), darkness (D), and pulsed lighting (PL). The NAL condition was presented first to all Ss, and the order of D and PL conditions was then counterbalanced across Ss. Thus, the NAL condition provided a baseline measurement from which each S's threshold shifts under D and PL conditions were measured.

In the NAL condition S's half of the suite was diffusely illuminated by means of four 40-w electric light bulbs in conical shields directed toward the ceiling. Illumination such as this, providing between 25 and $50 \mathrm{ft}-\mathrm{c}$, is typical in audiometric testing. A restricting visual-field box was used in both the D and PL conditions. The interior of the box was a 1-ft. cube; a hole was cut in one side to accommodate S's face. The edges of this oval hole were lined with 2-in. thick soft foam rubber, so that when $S$ pressed his head against it there was formed a light-tight, but comfortable seal with his eyes located slightly inside the box. The viewing screen (a 1-ft. square of light-diffusing ground glass) was on the opposite side of the box. The front surface of the glass was covered with a single layer of translucent tissue paper in the center of which a 1/4-in. black cross was placed as a fixation point. Except for the viewing screen, the interior of the box was painted a uniform flat white. In the $\mathrm{D}$ or darkness condition, there was no ambient light in S's half of the testing suite and an opaque black cloth was used to cover the entire box, including the screen and S's head; this further assured $E$ that no light would reach $S$ 's eyes.

In the PL or pulsed-light condition, the firing of the flourescent bulbs, which were mounted 6-in. behind the 
viewing screen, provided pulsed illumination of the screen and the interior of the visual-field box. In order to preclude any startle effect, $\mathrm{S}$ was allowed to adapt to the pulsed light for several seconds prior to the presentation of auditory stimuli.

The procedure and instructions were identical for all Ss. The instructions were those typically used in administering a Békésy audiometric examination. Emphasis was placed on the auditory task, and all other stimuli were identified as "irrelevant" to S.

Results

The mean threshold intensities obtained under the NAL condition are shown by the broken line in Fig. 1. The differences among the five test frequencies were statistically significant, as there was essentially no overlap.

Threshold shifts, obtained under the D and PL conditions relative to the NAL condition, were computed for each $S$ at each test frequency. The mean threshold shifts are shown by the solid lines in Fig. 1 (read from the ordinate on the right); a negative value indicates a lower threshold under the $\mathrm{D}$ or $\mathrm{PL}$ condition relative to the NAL condition. All except the D-500 and D-2000 points are negative.

An analysis of variance of the threshold-shift scores, based on the factorial design of two experimental conditions (D versus $P L$ ), five frequencies, and $20 \mathrm{Ss}$, resulted in the identification of only one significant source of variation. This was the condition-by-frequency interaction $(F=4.409, \mathrm{df}=4 / 76, \mathrm{p}<.005)$. In order to understand this interaction, two additional analyses were computed, one for each condition.

The analysis of the PL threshold shifts indicated that the differences among the frequencies were not significant (F-ratio less than unity). The differences among the $D$ threshold shifts were significant $(F=3.616, \mathrm{df}=$

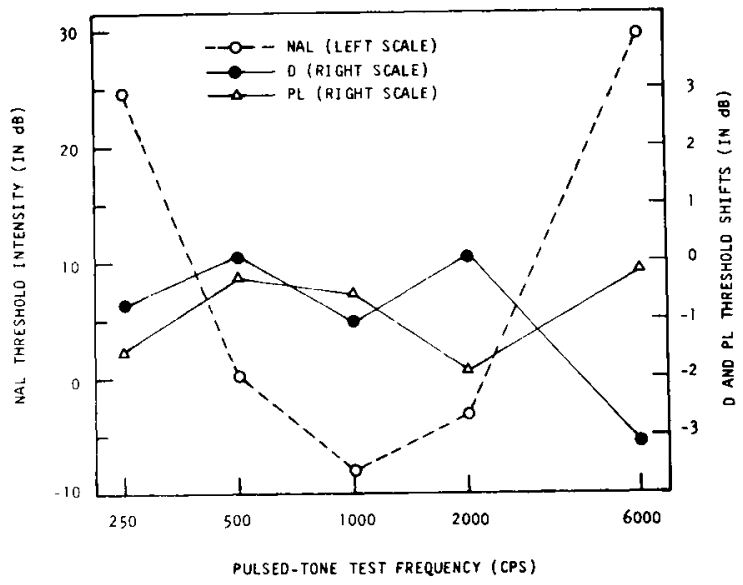

Fig. 1. Auditory threshold intensity for five pulsed-tone test frequencies under a baseline condition of nomal ambient lighting (NAL), and shifts in thresholds under conditions of darkness (D) and synchronized pulsed light (PL).
$4 / 76, p<.01)$. A Newman-Keuls test of all ordered pairs of means in the $\mathrm{D}$ condition indicated that the threshold at $6000 \mathrm{~Hz}$ was shifted to a significantly lower value than those at the other test frequencies $(p<.05$ in each case); no other difference was statistically significant.

\section{Discussion}

The results indicate that the threshold for a pulsed tone at $6000 \mathrm{~Hz}$ when obtained under conditions of total darkness was significantly lower than when obtained under conditions of either normal ambient illumination or synchronized pulsed light. This leads to the conclusion that the effect of concurrent visual stimulation on auditory sensitivity is dependent on frequency. There may be an effect at the higher frequencies $(6000 \mathrm{~Hz}$ and, perhaps, higher), whereas there is apparently no effect in the middle $(1000-2000 \mathrm{~Hz})$ or lower $(250-500 \mathrm{~Hz})$ ranges. This supports further the proposition that the three psycho-acoustic regions may represent different detection and coding processes in man (cf.Davis, 1961).

Since the $6000-\mathrm{Hz}$ threshold was lower in darkness, it must be concluded that auditory sensitivity was impaired by concurrent visual stimulation. This suggests that audio-visual interactions are intransitive-i.e., that concurrent auditory stimulation will improve visual sensitivity, whereas concurrent visual stimulation will impair auditory sensitivity in the higher frequency ranges.

\section{References}

Davis, H. Peripheral coding of auditory information. In W. A. Rosenblith (Ed.), Sensory communication. Cambridge, Mass.: M. I. T. Press, 1961.

Gregg, L. W., \& Brogden, W. J. Auditory sensitivity. J. exp. Psy chol., 1952, 43, 179-186.

Gulick, W. L., \& Smith, F. L. The effect of intensity of visual stimulation upon auditory acuity. Psychol. Rec., 1959, 9, 29-32.

Markov, P. $\mathbf{O}$. The problem of the influence of extremely intense optic stimulation on the visual, auditory, and cutaneous analyzers in man. Biofizika, 1960, 5, 677-684 (Abstract).

Moruyama, K. Contralateral relationship between the ears and the halves of visual field in the sensory interaction. Tokaku psychol. Folia, 1961, 19,81-92.

Rubinstein, M. K., \& Loeb, M. Interaction between vision and audition. U. S. Army Med. Res. Lab. Rep., 1955, No. 151.

Smith, W. M. Visual recognition: Facilitation of seeing by hearing. Psychon. Sci, 1965, 2, 157-158.

Symonds, J. R. The effect of various heteromodal stimuli on visual sensitivity. Quart. J. exp. Psychol., 1963, 15, 243-251.

Thompson, R. F., Voss, J. F., \& Brogden, W. J. Effect of brightness of simultaneous visual stimulation on absolute auditory sensitivity. J. exp. Psychol., 1958, 55, 45-50.

\section{Notes}

1. Supported in part by the U. S. Army Medical Research and Development Command, Department of the Army, under Research Contract No. DA-49-193-MD-2567, "Behavioral Effects of Infectious Diseases." Equipment and facilities of the Audiology and Speech Pathology Service, Veterans Administration Regional Office, Louisville, Ky., were made available for the collection of the data of this study; without this assistance, the study could not have been completed.

2. Now at Bell Telephone Laboratories, Murray Hill, N. J.

3. Now at University of Buffalo.

4. Also, Chief, Audiology and Speech Pathology Service VARO, Louisville. 